Trab-571-99 (9 páginas)

\title{
FLORÍSTICA ARBÓREA DA MATA DA PEDREIRA, MUNICÍPIO DE VIÇOSA, MINAS GERAIS ${ }^{1}$
}

\author{
Luiz Carlos Marangon², João Juares Soares ${ }^{3}$ e Ana Lícia Patriota Feliciano ${ }^{2}$
}

\begin{abstract}
RESUMO - O local de estudo é uma mata secundária, com área de 39,81 ha, localizada no município de Viçosa, Zona da Mata do Estado de Minas Gerais, nas coordenadas de $20^{\circ} 45^{\prime} \mathrm{S}$ e $42^{\circ} 55^{\prime} \mathrm{W}$, e $680 \mathrm{~m}$ de altitude. A temperatura média anual da região varia de 14,6 a $21,8^{\circ} \mathrm{C}$ e a precipitação média anual é de $1.220 \mathrm{~mm}$. A vegetação caracteriza como Floresta Estacional Semidecidual. No levantamento florístico realizado observaram-se 197 espécies, distribuídas em 53 famílias botânicas e 134 gêneros. A florística encontrada foi comparada de forma qualitativa à de outros trabalhos da flora arbórea realizados nas Regiões Sudeste e Sul do Brasil.
\end{abstract}

Palavras-chave: Florística arbórea e floresta estacional semidecidual.

\section{MATA DA PEDREIRA ARBOREAL FLORISTICS, VIÇOSA, MINAS GERAIS, BRAZIL}

\begin{abstract}
The area studied is a secondary forest of approximately 40 ha, located in Viçosa, Zona da Mata Minas Gerais at 20 $45^{\prime} \mathrm{S}$ and $42^{\circ} 55^{\prime} \mathrm{W}$ coordinates and $680 \mathrm{~m}$ altitude. The mean annual temperature ranges from $14.6{ }^{\circ} \mathrm{C}$ to $21.8{ }^{\circ} \mathrm{C}$ and the mean annual precipitation is $1.220 \mathrm{~mm}$. The vegetation is classified as Semideciduous Seasonal Forest. The floristic survey carried out in the area reported 197 species distributed among 53 families and 134 genera. The floristcs found was qualitatively compared with other arboreal flora works carried out in the southeast and south of Brazil.
\end{abstract}

Key words: $\quad$ Arboreal floristics and semideciduous seasonal forest.

\section{INTRODUÇÃO}

As fitofisionomias de Minas Gerais predominavam em uma vasta região correspondente ao centro-sul e ao leste do Estado. A formação vegetal que sugeriu o nome Zona da Mata formava um contínuo com a floresta do médio Paraíba, ao sul, e a do vale do Rio Doce, ao norte. A oeste, limitavam-na os campos naturais do centro e do sul de Minas (Valverde, 1958). Essa afirmativa vem confirmar a presença das florestas densas e contínuas existentes há menos de um século, na região conhecida como "área da mata", na qual a formação florestal predominante é a Floresta Estacional Semidecidual (Veloso \& Goes-Filho, 1982).
O conhecimento e o entendimento da complexa dinâmica que envolve as florestas tropicais iniciam-se pelo levantamento da florística. A identidade das espécies e o seu comportamento em comunidades vegetais são o começo de todo processo para compreensão deste ecossistema.

A Zona da Mata mineira necessita de estudos básicos de vegetação, pois ela não está em situação diferente das demais regiões do País, sofrendo alto grau de perturbação decorrente de práticas agrícolas inadequadas e da expansão demográfica, mineradora e industrial. Neste sentido, estudos recentes vêm contribuindo a cada dia para o conhecimento da flora arbórea dessa região, como os de

1 Recebido para publicação em 28.10.2001.

Aceito para publicação em 19.2.2003.

2 Departamento de Ciência Florestal da Universidade Federal Rural de Pernambuco - UFRPE. ${ }^{3}$ Departamento de Botânica da Universidade Federal de São Carlos - UFSCar/PPG-ERN. 
Mariscal Flores (1993), Meira-Neto et al. (1997) e Almeida (1996), realizados na tentativa de preservar os remanescentes florestais da referida região.

As Florestas Semideciduais do Estado de Minas Gerais sofrem o mesmo processo de perturbação dos demais ecossistemas brasileiros. Os poucos nativos remanescentes necessitam urgentemente de pesquisas básicas, no sentido de promover a conservação e a preservação dos fragmentos florestais da região.

Portanto, o objetivo deste trabalho foi estudar a flora arbórea da Mata da Pedreira, no sentido de conhecer as espécies desta comunidade, como também de estabelecer uma base para promover o manejo sustentado para esta região da Zona da Mata de Minas Gerais.

\section{MATERIAL E MÉTODOS}

\subsection{Caracterização da Área de Estudo}

A Mata da Pedreira (Figura 1) localiza-se na Estação Experimental Mata do Paraíso, no município de Viçosa, a $6 \mathrm{~km}$ da cidade, nas coordenadas $20^{\circ} 45^{\prime} \mathrm{S}$ e $42^{\circ} 55^{\prime} \mathrm{W}$. Possui uma área de 39,81 ha e está ao norte da Zona da Mata mineira. A altitude varia de 600 a 800 m, podendo ser encontradas altitudes superiores a $800 \mathrm{~m}$ nos topos dos morros da região (Meira-Neto, 1997). A área estudada apresenta, em termos de altitude, uma cota mínima de 730 m e uma cota máxima de $870 \mathrm{~m}$. A mata é secundária, preservada desde 1965. Fotos aéreas do local na década de 60 mostram pastagens e pequenas áreas com capoeiras baixas, além da pedreira que era explorada naquela época.

\subsubsection{Clima}

Segundo a classificação de Köpen, o clima é do tipo Cwb, mesotérmico, com verões chuvosos, invernos frios e secos. A precipitação média nos últimos 30 anos foi de cerca de $1.221 \mathrm{~mm}$. Há um excedente hídrico de novembro a abril; a precipitação fica abaixo da evapotranspiração potencial de abril até setembro, causando um déficit hídrico nesse período; e nos meses de setembro a novembro a precipitação volta a ser maior que a evapotranspiração, na qual se define uma estação seca e outra chuvosa na região de Viçosa-MG (Marangon, 1999).

\subsubsection{Geologia e Relevo}

A área é gnáissica e a rocha é mesocrática bastante lineada, sendo a geologia pertencente ao Pré-cambriano D
(Pré-cambriano inferior ou indiviso), segundo Resende (1971).

Quanto à topografia, são bem característicos os chamados mares de morros, de ampla distribuição na paisagem, cujos vales estreitos apresentam relevo acidentado, ondulado e montanhoso.

\subsubsection{Solo}

Nos topos e nas encostas dos morros ocorre o Latossolo Vermelho-Amarelo álico, enquanto nos terraços o solo é o Podzólico Vermelho-Amarelo Câmbico (Resende \& Correa, citados por Meira-Neto 1997).

\subsubsection{Vegetação}

A formação florestal predominante na região é a Floresta Estacional Semidecidual, que pode ser ainda submontana e montana. A submontana ocorre freqüentemente nas encostas interioranas das Serras da Mantiqueira e dos Órgãos, nos planaltos centrais capeados pelos arenitos Botucatu, Bauru e Caiuá, dos períodos geológicos Jurássico e Cretáceo. Distribui-se desde o Espírito Santo e sul da Bahia até o Rio de Janeiro, Minas Gerais, São Paulo, sudoeste do Paraná e sul do Mato Grosso do Sul. Os gêneros dominantes, com indivíduos deciduais, são os mesmos que ocorrem na floresta ombrófila atlântica, como: Cedrela, Parapiptadenia e Cariniana; nos planaltos areníticos, os ecótipos deciduais que caracterizam esta formação pertencem aos gêneros amazônicos Hymenaea, Copaifera, Peltophorum, Astronium, Tabebuia, Balfourodendron e muitos outros. A montana, onde as formações estabelecidas estão acima de $500 \mathrm{~m}$ de altitude, situa-se principalmente na face interior da Serra dos Órgãos, no Estado do Rio de Janeiro, e na Serra da Mantiqueira, nos Estados de São Paulo, Rio de Janeiro, Minas Gerais e Espírito Santo. A formação montana é quase sempre dominada pelo gênero Anadenanthera, de origem amazônica (Veloso et al., 1991).

\subsection{Levantamento Florístico}

Com o intuito de conseguir uma caracterização da flora arbórea do local, foram realizadas campanhas de campo até cobrir toda área de estudo. Foram feitas coletas de material vegetativo e reprodutivo presentes nas árvores, ou no chão, durante um ano (de 1996 a 1997). 
Para as coletas, utilizaram-se tesoura de alta poda e poda manual. O material botânico foi levado para o setor de Dendrologia do Departamento de Engenharia Florestal da Universidade Federal de Viçosa, onde foi devidamente preparado e, posteriormente, identificado. Todo material coletado foi incorporado à coleção de exsicatas do referido setor. A coleta do material botânico seguiu as determinações de Vieira \& Carvalho-Okano (1985) e foi identificado em termos de família, gênero e espécie. Para isto, utilizaram-se referências de Correa (1929), Rizzini (1971), Reitz et al. (1979), Barroso et al. (1984), Lorenzi (1992) e publicações especializadas. Nas dúvidas de identificação, recorreu-se ao herbário VIC do Departamento de Biologia Vegetal da Universidade Federal de Viçosa.

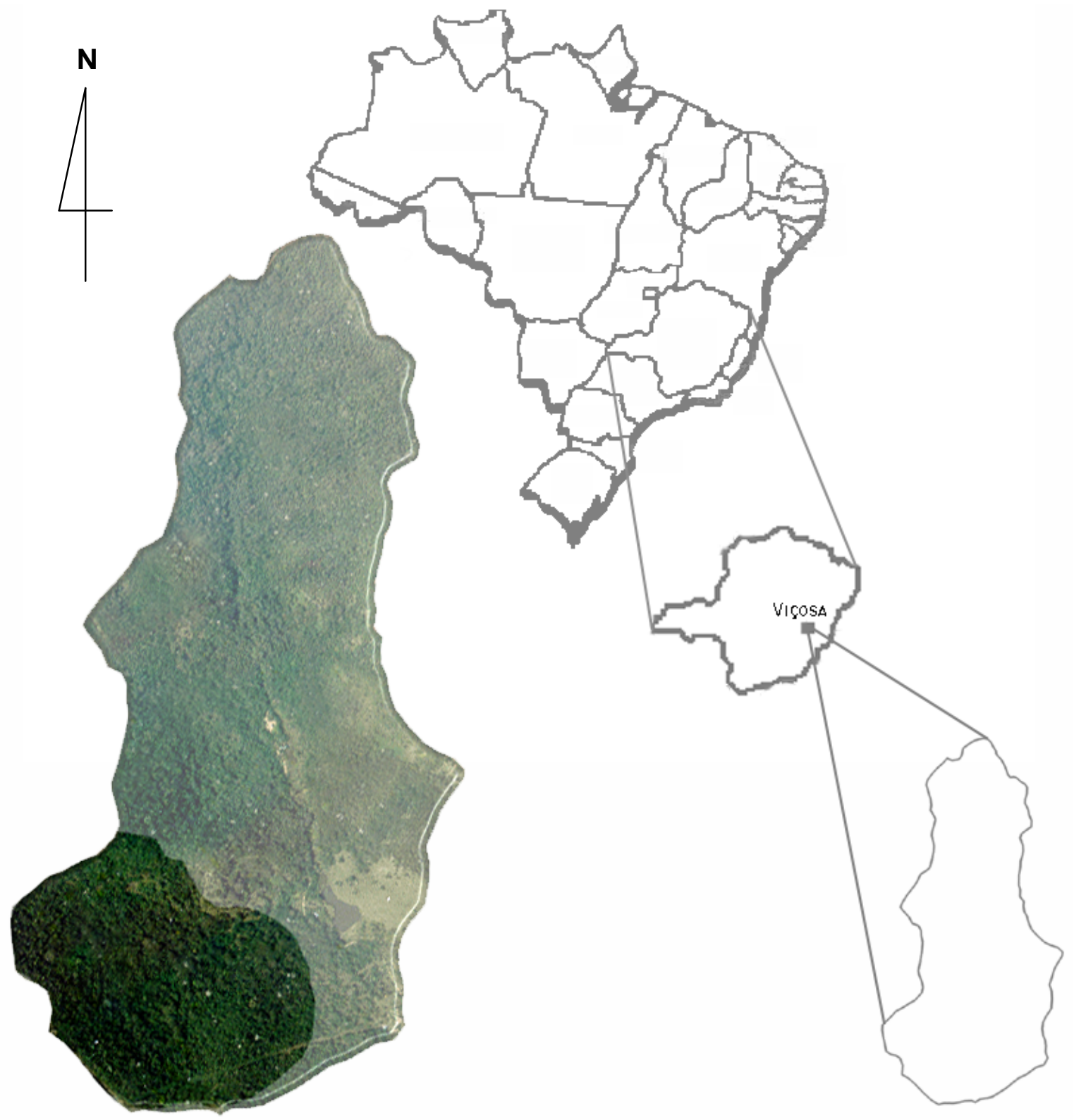

Figura 1 - Localização geográfica da Estação Experimental Mata do Paraíso, com destaque para a Mata da Pedreira, Viçosa-MG. Figure 1 - Geographic location of the Experimental Station Mata do Paraíso, with empahasis on Mata da Pedreira, Viçosa-MG, Brazil. 
$\mathrm{Na}$ classificação das espécies usou-se o sistema Cronquist (1988) e na grafia dos binômios, com seus respectivos autores, recorreu-se ao índice de espécies do Royal Botanic Garden (1993).

Para a composição florística, no que diz respeito à similaridade, comparações foram feitas com florestas estudadas em Minas Gerais e em outros Estados do Sudeste e Sul do Brasil. Para tal procedimento, utilizouse o índice de similaridade de Sorensen (MuellerDombois \& Ellemberger, 1974). Os trabalhos usados com esta finalidade foram: Silva \& Leitão Filho (1982), Pagano et al. (1987), Oliveira-Filho \& Machado (1993), Oliveira-Filho et al. (1994), Negrelle (1995), Meira-Neto (1997), Salomão (1998) e Lopes (1998).

\section{RESULTADOS E DISCUSSÃO}

No levantamento florístico da área de estudo foram encontradas 197 espécies, 53 famílias e 134 gêneros. Tais resultados encontram-se no Quadro 1.

Os gêneros ocorreram da seguinte forma: Eugenia, com 6 espécies; Solanum e Casearia, com 5; Guarea, Inga, Machaerium, Nectandra, Ocotea e Trichilia, com 4; Aspidosperma e Croton, com 3; e Alchornea, Allophylus, Anadenanthera, Andira, Annona, Bauhinia, Cariniana, Cecropia, Cordia, Erythroxylum, Erythrina, Ficus, Guatteria, Lamanonia, Matayba, Maytenus, Miconia, Myrcia, Myrciaria, Piptadenia, Pouteria, Psychotria, Senna, Swartzia, Vernonia, Vitex, Xylopia e Zanthoxylum, com 2 espécies. Os demais, 95 gêneros, apresentaram uma única espécie.

As famílias presentes na área de estudo, em termos de diversidade de espécies, ficaram assim distribuídas: Leguminosae Papilionoideae, com 15 espécies; Leguminosae Caesalpinioideae, com 13; Myrtaceae, com 11; Euphorbiaceae, Lauraceae, Meliaceae, Leguminosae Mimosoideae e Rubiaceae, com 10; Annonaceae e Flacourtiaceae, com 7; Sapindaceae e Solanaceae, com 6; Apocynaceae, Bignoniaceae e Moraceae, com 5; Rutaceae e Verbenaceae, com 4; Anacardiaceae, Arecaceae, Asteraceae, Bombacaceae e Melastomataceae, com 3; e Boraginaceae, Cecropiaceae, Celastraceae, Cunoniaceae, Erythroxylaceae, Guttiferae, Lecythidaceae, Monimiaceae, Myrsinaceae, Rhamnaceae e Vochysiaceae, com 2. As 20 famílias restantes apresentaram uma única espécie.

Resultados semelhantes foram encontrados por Meira-Neto (1997), em um fragmento de Floresta Estacional Semidecidual (Mata da Silvicultura) de 17 ha, no município de Viçosa-MG, onde foram relacionadas 154 espécies arbóreas, 105 gêneros e 47 famílias botânicas. Quanto à riqueza, as famílias que mais se destacaram foram: Rubiaceae, Fabaceae (Leguminosae Papilionoideae), Lauraceae, Mimosaceae (Leguminosae Mimosoideae), Euphorbiaceae, Myrtaceae, Annonaceae, Flacourtiaceae e Melastomataceae. A Mata da Pedreira também apresenta diversidade muito próxima daquela encontrada por LeitãoFilho (1982), no que diz respeito às espécies por famílias botânicas, em áreas de Florestas Estacionais Semideciduais no Estado de São Paulo, onde se destaca a riqueza das famílias Fabaceae (Leguminosae Papilionoideae, Euphorbiaceae, Myrtaceae, Lauraceae e Rubiaceae. Tal semelhança foi também verificada por Lopes (1998), estudando a região do Vinhático, no Parque Estadual do Rio Doce, em Minas Gerais, onde a vegetação também é Floresta Estacional Semidecidual e a diversidade por famílias botânicas, em especial, foram Fabaceae (Leguminosae Papilionoideae), Mimosaceae (Leguminosae Mimosoideae), Lauraceae, Myrtaceae, Euphorbiaceae, Caesalpiniaceae (Leguminosae Caesalpinioideae) e Annonaceae. Tal autor relatou que as famílias encontradas confirmaram-se como de maior riqueza específica em estudos realizados por Pagano et al. (1987), Oliveira-Filho \& Machado (1993) e Oliveira- Filho et al. (1994). Quando o objetivo foi estabelecer a similaridade florística entre a Mata da Pedreira e os estudos fitossociológicos realizados em Floresta Estacional Semidecidual e Floresta Ombrófila Densa (Veloso et al., 1991), verificou-se que a área de estudo apresentou maior ligação com a Mata da Silvicultura (MeiraNeto, 1997), cujo índice de similaridade foi de $47,17 \%$ (Quadro 2), e com as áreas estudadas na região do Vinhático, no Parque Florestal do Rio Doce-MG (Lopes, 1998), em Tiradentes-MG (Oliveira- Filho \& Machado, 1993) e em Rio Claro-SP (Pagano et al., 1987), no qual os índices de similaridade foram de $33,56,32,62$ e $28,73 \%$, respectivamente (Quadro 2). Tais formações florestais estudadas são Florestas Estacionais Semideciduais.

A menor ligação foi com as áreas de Lavras, Estado de Minas Gerais (Oliveira-Filho et al., 1994), com 12,03\% (Quadro 2), cuja tipologia é Floresta Estacional Semidecidual, e as áreas da Floresta Nacional do Rio Preto, Estado do Espírito Santo (Salomão, 1998), com 12,37\% (Quadro 2) e Volta Velha, Estado de Santa Catarina (Negrelle, 1995), com 11,07\% (Quadro 2). As duas últimas áreas correspondem à Floresta Ombrófila Densa. A similaridade mais baixa foi a área de Ubatuba, Estado de São Paulo (Silva \& Leitão-Filho, 1982), que apresentou ligação de 7,40\% (Quadro 2) e está localizada em uma Floresta Ombrófila Densa. 
Quadro 1 - Listagem das espécies arbóreas, em ordem alfabética, de família, de gênero e de espécies encontradas na Mata da Pedreira, no município de Viçosa, Minas Gerais

Table 1 - List of arboreal species in alphabetical order of family, genus and species of Pedreira Forest, Viçosa, Minas Gerais, Brazil

\begin{tabular}{|c|c|}
\hline Família/Espécie & Coleta \\
\hline $\begin{array}{l}\text { ANACARDIACEAE } \\
\text { Astronium fraxinifolium Schott } \\
\text { Schinus terebinthifolius Raddi } \\
\text { Tapirira guianensis Aubl. } \\
\end{array}$ & $\begin{array}{l}2883 \\
2930 \\
2891\end{array}$ \\
\hline $\begin{array}{l}\text { ANNONACEAE } \\
\text { Annona cacans } \text { Warm. } \\
\text { Annona } \text { sp. } \\
\text { Guatteria latifolia (Mart.) R. E. Fries } \\
\text { Guatteria villosissima A. St.-Hil. } \\
\text { Rollinia silvatica } \text { Mart. } \\
\text { Xylopia brasiliensis } \text { Spreng. } \\
\text { Xylopia sericea A. St.-Hil. } \\
\end{array}$ & $\begin{array}{l}2890 \\
2892 \\
2884 \\
2885 \\
2886 \\
2901 \\
2900 \\
\end{array}$ \\
\hline $\begin{array}{l}\text { APOCYNACEAE } \\
\text { Aspidosperma cylindrocarpum Müll. Arg. } \\
\text { Aspidosperma parvifolium A. DC. } \\
\text { Aspidosperma polyneuron Müll. Arg. } \\
\text { Himatanthus phagedaenicus (Mart.) Woodson } \\
\text { Taberbaemontana hystrix (Steud.) A.DC. }\end{array}$ & $\begin{array}{l}2929 \\
2933 \\
2934 \\
2941 \\
2940\end{array}$ \\
\hline $\begin{array}{l}\text { AQUIFOLIACEAE } \\
\text { Ilex cerasifolia } \text { Reissek }\end{array}$ & 2960 \\
\hline $\begin{array}{l}\text { ARALIACEAE } \\
\text { Schefflera morototoni (Aubl.) Maguire, Steyerm. \& } \\
\text { Frodin }\end{array}$ & 2965 \\
\hline $\begin{array}{l}\text { ARECACEAE } \\
\text { Attalea dubia Burret } \\
\text { Euterpe edulis Mart. } \\
\text { Syagrus romanzoffiana (Cham.) Glassm. } \\
\end{array}$ & $\begin{array}{l}2951 \\
2969 \\
2990\end{array}$ \\
\hline $\begin{array}{l}\text { ASTERACEAE } \\
\text { Piptocarpha macropoda } \text { Baker } \\
\text { Vernonia diffusa Less. } \\
\text { Vernonia discolor Less. } \\
\end{array}$ & $\begin{array}{l}2887 \\
2888 \\
2894\end{array}$ \\
\hline $\begin{array}{l}\text { BIGNONIACEAE } \\
\text { Cybistax antisyphilitica Mart. } \\
\text { Jacaranda macrantha Cham. } \\
\text { Sparattosperma leucanthum K. Schum. } \\
\text { Tabebuia chrysotricha (Mart. ex DC.) Standl. } \\
\text { Zeyheria tuberculosa Bureau ex Verl. }\end{array}$ & $\begin{array}{l}3070 \\
3052 \\
2893 \\
2897 \\
2899\end{array}$ \\
\hline $\begin{array}{l}\text { BOMBACACEAE } \\
\text { Ceiba speciosa (A. St.-Hil.) Gibbs \& Semir } \\
\text { Eriotheca candolleana (K. Schum.) A. Robyns } \\
\text { Pseudobombax grandiflorum (Cav.) A. Robyns } \\
\end{array}$ & $\begin{array}{l}2898 \\
3021 \\
3022 \\
\end{array}$ \\
\hline $\begin{array}{l}\text { BORAGINACEAE } \\
\text { Cordia sericicalyx A. DC. } \\
\text { Cordia trichotoma } \text { Vell. ex Steud. }\end{array}$ & $\begin{array}{l}3000 \\
2946 \\
\end{array}$ \\
\hline $\begin{array}{l}\text { BURSERACEAE } \\
\text { Trattinickia ferruginea Kuhlm. }\end{array}$ & 2910 \\
\hline $\begin{array}{l}\text { CECROPIACEAE } \\
\text { Cecropia glaziovi Snethl. } \\
\text { Cecropia hololeuca Miq. }\end{array}$ & $\begin{array}{l}2911 \\
2915 \\
\end{array}$ \\
\hline $\begin{array}{l}\text { CELASTRACEAE } \\
\text { Maytenus aquifolium Mart. } \\
\text { Maytenus robusta } \text { Reissek }\end{array}$ & $\begin{array}{l}3004 \\
3006\end{array}$ \\
\hline
\end{tabular}

Continua...

Continued...
Quadro 1, cont.

Table 1, cont.

\begin{tabular}{|c|c|}
\hline Família/Espécie & Coleta \\
\hline $\begin{array}{r}\text { CHRYSOBALANACEAE } \\
\text { Hirtella selloana Hook.f. }\end{array}$ & 3013 \\
\hline $\begin{array}{l}\text { COMBRETACEAE } \\
\text { Terminalia brasiliensis Eichl. }\end{array}$ & 3035 \\
\hline $\begin{array}{l}\text { CLETHRACEAE } \\
\text { Clethra scabra } \text { Pers. }\end{array}$ & 3045 \\
\hline $\begin{array}{l}\text { CUNONIACEAE } \\
\text { Lamanonia tomentosa Kuntze } \\
\text { Lamanonia glabra Kuntze }\end{array}$ & $\begin{array}{l}3053 \\
3060\end{array}$ \\
\hline $\begin{array}{l}\text { ERYTHROXYLACEAE } \\
\text { Erythroxylum pelleterianum A. St.-Hil. } \\
\text { Erythroxylum sp. }\end{array}$ & $\begin{array}{l}3061 \\
3062 \\
\end{array}$ \\
\hline $\begin{array}{l}\text { EUPHORBIACEAE } \\
\text { Alchornea glandulosa Poepp. \& Endl. } \\
\text { Alchornea triplinervia Müll. Arg. } \\
\text { Croton floribundus Spreng. } \\
\text { Croton priscus Croizat } \\
\text { Croton urucurana Baill. } \\
\text { Hieronyma alchorneoides Allemão } \\
\text { Mabea fistulifera Mart. } \\
\text { Manihot pilosa Pohl } \\
\text { Maprounea guianensis Aubl. } \\
\text { Sapium glandulatum (Vell.) Pax }\end{array}$ & $\begin{array}{l}3069 \\
3066 \\
3064 \\
2999 \\
2991 \\
2997 \\
2992 \\
2895 \\
2896 \\
2903\end{array}$ \\
\hline $\begin{array}{l}\text { FLACOURTIACEAE } \\
\text { Carpotroche brasiliensis Endl. } \\
\text { Casearia aculeata Jacq. } \\
\text { Casearia arborea Urb. } \\
\text { Casearia decandra Jacq. } \\
\text { Casearia gossypiosperma Briq. } \\
\text { Casearia sylvestris Sw. } \\
\text { Xylosma prockia (Turcz.) Turcz. }\end{array}$ & $\begin{array}{l}2925 \\
2916 \\
2936 \\
2984 \\
2988 \\
2975 \\
2993 \\
\end{array}$ \\
\hline $\begin{array}{l}\text { GUTTIFERAE } \\
\text { Garcinia spruceana Engl. } \\
\text { Vismia guianensis (Aubl.) Choisy }\end{array}$ & $\begin{array}{l}2976 \\
2914 \\
\end{array}$ \\
\hline $\begin{array}{l}\text { LABIATAE } \\
\text { Hyptidendron asperrimum ( Spreng.) Harley }\end{array}$ & 2986 \\
\hline $\begin{array}{l}\text { LACISTEMACEAE } \\
\text { Lacistema pubescens Mart. }\end{array}$ & 2968 \\
\hline $\begin{array}{l}\text { LAURACEAE } \\
\text { Endlicheria paniculata (Spreng.) J.F. Macbr. } \\
\text { Nectandra lanceolata Nees \& Mart. ex Nees } \\
\text { Nectandra reticulata Mez } \\
\text { Nectandra rigida } \text { Nees } \\
\text { Nectandra megapotamica (Spreng.) Mez } \\
\text { Ocotea corymbosa } \mathrm{Mez} \\
\text { Ocotea laxa } \mathrm{Mez} \\
\text { Ocotea martiana } \mathrm{Mez} \\
\text { Ocotea odorifera } \text { (Vell.) Rohwer } \\
\text { Persea pyrifolia } \text { Nees }\end{array}$ & $\begin{array}{l}2966 \\
3015 \\
3018 \\
3030 \\
3039 \\
3050 \\
3048 \\
3063 \\
3056 \\
2943 \\
\end{array}$ \\
\hline $\begin{array}{l}\text { LECYTHIDACEAE } \\
\text { Cariniana estrellensis Kuntze } \\
\text { Cariniana legalis Kuntze }\end{array}$ & $\begin{array}{l}2937 \\
2949\end{array}$ \\
\hline
\end{tabular}


Quadro 1, cont.

Table 1, cont.

\begin{tabular}{|c|c|}
\hline \multirow{2}{*}{\multicolumn{2}{|c|}{$\begin{array}{c}\text { Família/Espécie } \\
\end{array}$}} \\
\hline & \\
\hline Apuleia leiocarpa J.F. Macbr. & 2908 \\
\hline Bauhinia forficata Link & 3001 \\
\hline Bauhinia sp. & 3002 \\
\hline Cassia ferruginea Schrad. ex DC. & 3024 \\
\hline Copaifera langsdorffii Desf. & 3026 \\
\hline Hymenaea courbaril L. var. stilbocarpa (Hayne) Y.T. & 3044 \\
\hline Lee et Langenh. & \\
\hline Melanoxylon brauna Schott & 3033 \\
\hline Schizolobium parahyba (Vell.) Blake & 2889 \\
\hline Sclerobium rugosum Mart. & 3031 \\
\hline Senna macranthera (DC. ex Collad.) Irwin. \&.Barneby & 3009 \\
\hline Senna multijuga (L. C. Rich.) Irwin \& Barneby & 3005 \\
\hline Swartzia langsdorffii Raddi & 2921 \\
\hline Swartzia myrtifolia $\mathrm{Sm}$. & 2902 \\
\hline \multicolumn{2}{|l|}{ LEGUMINOSAE MIMOSOIDEAE } \\
\hline Albizia polycephala (Benth.) Killip & 2998 \\
\hline Anadenanthera colubrina (Vell.) Brenan & 3003 \\
\hline Anadenanthera peregrina Speg. & 3041 \\
\hline Dimorphandra mollis Benth. & 2906 \\
\hline Inga vera Willd. subsp. affinis (DC.) T. D. Penn. & 3067 \\
\hline Inga cylindrica Mart. & 3046 \\
\hline Inga edulis Mart. & 3029 \\
\hline Inga laurina $(\mathrm{Sw}$.$) Willd$ & 2909 \\
\hline Piptadenia adiantoides J.F. Macbr. & 2991 \\
\hline Piptadenia gonoacantha J.F. Macbr. & 2801 \\
\hline \multicolumn{2}{|l|}{ LEGUMINOSAE PAPILIONOIDEAE } \\
\hline Andira anthelmia (Vell.) J.F. Macbr. & 2913 \\
\hline Andira fraxinifolia Benth. & 2955 \\
\hline Bowdichia virgilioides H.B.K & 2959 \\
\hline Dalbergia nigra Allemão ex Benth. & 2907 \\
\hline Erythrina falcata Benth. & 2920 \\
\hline Erythrina verna Vell. & 2995 \\
\hline Lonchocarpus muehlbergianus Hassl. & 2996 \\
\hline Machaerium aculeatum Raddi & 3055 \\
\hline Machaerium & 3036 \\
\hline Machaerium stipitatum Vogel & 3016 \\
\hline Machaerium triste Vogel & 3020 \\
\hline Platymiscium pubescens Micheli & 3012 \\
\hline Platycyamus regnellii Benth. & 3011 \\
\hline Platypodium elegans Vogel & 2904 \\
\hline Zollernia ilicifolia Vogel & 2920 \\
\hline \multicolumn{2}{|l|}{ MELASTOMATACEAE } \\
\hline Miconia cinnamomifolia Triana & 2905 \\
\hline Miconia pusilliflora Triana & 2919 \\
\hline Tibouchina granulosa Cogn. & 2954 \\
\hline \multicolumn{2}{|l|}{ MELIACEAE } \\
\hline Cabralea canjerana (Vell.) Mart. & 2963 \\
\hline Cedrela fissilis Vell. & 3008 \\
\hline Guarea guidonia (L.) Sleumer & 3043 \\
\hline Guarea kunthiana A. Juss. & 2980 \\
\hline $\begin{array}{l}\text { Guarea macrophylla Vahl ssp. tuberculata (Vell.) T.D. } \\
\text { Penn. }\end{array}$ & 2994 \\
\hline Guarea pendula Ramalho, Pinheiro \& T.D. Penn. & 3068 \\
\hline Trichilia catigua A. Juss. & 3058 \\
\hline Trichilia hirta $\mathrm{L}$. & 3023 \\
\hline Trichilia lepidota $\mathrm{Sw}$. & 2971 \\
\hline Trichilia pallida $\mathrm{Sw}$. & 2982 \\
\hline
\end{tabular}

Continua...

Continued...
Quadro 1, cont.

Table 1, cont.

\begin{tabular}{|c|c|}
\hline Família/Espécie & Coleta \\
\hline $\begin{array}{l}\text { MONIMIACEAE } \\
\text { Mollinedia floribunda Tul. } \\
\text { Siparuna arianeae V. Pereira }\end{array}$ & $\begin{array}{l}2947 \\
2953\end{array}$ \\
\hline $\begin{array}{l}\text { MORACEAE } \\
\text { Brosimum guianense Huber ex Ducke } \\
\text { Ficus glabra Vell. } \\
\text { Ficus insipida Willd. } \\
\text { Maclura tinctoria D. Don ex Steud. } \\
\text { Sorocea guilleminiana Gaudich. } \\
\end{array}$ & $\begin{array}{l}2964 \\
3007 \\
2932 \\
2912 \\
2972 \\
\end{array}$ \\
\hline $\begin{array}{l}\text { MYRISTICACEAE } \\
\text { Virola oleifera (Schott) A. C. Sm. }\end{array}$ & 2981 \\
\hline $\begin{array}{l}\text { MYRSINACEAE } \\
\text { Cybianthus indecorus Mez } \\
\text { Myrsine coriacea }(\mathrm{Sw} .) \mathrm{R} . \mathrm{Br} \text {. }\end{array}$ & $\begin{array}{l}3014 \\
3025 \\
\end{array}$ \\
\hline $\begin{array}{l}\text { MYRTACEAE } \\
\text { Campomanesia xanthocarpa } \text { Berg } \\
\text { Eugenia dodonaefolia Cambess. } \\
\text { Eugenia florida DC. } \\
\text { Eugenia leptoclada } \text { Berg } \\
\text { Eugenia speciosa Cambess. } \\
\text { Eugenia diantha } \text { Berg } \\
\text { Eugenia sp. } \\
\text { Myrcia rostrata } \text { DC. } \\
\text { Myrcia rufula Miq. } \\
\text { Myrciaria floribunda Berg. } \\
\text { Myrciaria sp. } \\
\end{array}$ & $\begin{array}{l}3032 \\
3065 \\
2997 \\
2989 \\
2935 \\
3040 \\
2950 \\
2961 \\
2918 \\
3028 \\
3042 \\
\end{array}$ \\
\hline $\begin{array}{l}\text { NYCTAGINACEAE } \\
\text { Guapira opposita (Vell.) Reitz }\end{array}$ & 2917 \\
\hline $\begin{array}{l}\text { OCHNACEAE } \\
\text { Ouratea polygyna Engl. }\end{array}$ & 2952 \\
\hline $\begin{array}{l}\text { OPILIACEAE } \\
\text { Agonandra englerii Hoehne }\end{array}$ & 3038 \\
\hline $\begin{array}{l}\text { PHYTOLACCACEAE } \\
\text { Seguieria langsdorffii Moq. }\end{array}$ & 2957 \\
\hline $\begin{array}{l}\text { PROTEACEAE } \\
\text { Roupala brasiliensis } \text { Klotzsch }\end{array}$ & 2922 \\
\hline $\begin{array}{l}\text { RHAMNACEAE } \\
\text { Colubrina glandulosa Perkins } \\
\text { Rhamnidium elaeocarpum Reissek }\end{array}$ & $\begin{array}{l}3034 \\
2934 \\
\end{array}$ \\
\hline $\begin{array}{l}\text { ROSACEAE } \\
\text { Prunus sellowii Koehne }\end{array}$ & 2958 \\
\hline $\begin{array}{l}\text { RUBIACEAE } \\
\text { Alibertia sp. } \\
\text { Amaioua guianensis Aubl. } \\
\text { Bathysa nicholsonii K. Schum. } \\
\text { Coutarea speciosa Aubl. } \\
\text { Faramea multiflora A. Rich. } \\
\text { Guettarda viburnoides Cham. \& Schltdl. } \\
\text { Ixora warmingii Müll. Arg. } \\
\text { Psychotria carthagenensis Jacq. } \\
\text { Psychotria sessilis (Vell.) Müll. Arg. } \\
\text { Randia nitida (Kunth) DC. } \\
\end{array}$ & $\begin{array}{l}2923 \\
2938 \\
3059 \\
2939 \\
2926 \\
2970 \\
3054 \\
3017 \\
2974 \\
2985 \\
\end{array}$ \\
\hline $\begin{array}{l}\text { RUTACEAE } \\
\text { Dictyoloma vandellianum A. Juss. } \\
\text { Hortia arborea Engl. } \\
\text { Zanthoxylum rhoifolium Lam. } \\
\text { Zanthoxylum riedelianum Engl. }\end{array}$ & $\begin{array}{l}2978 \\
3019 \\
2924 \\
2945\end{array}$ \\
\hline
\end{tabular}

Continua...

Continued... 
Quadro 1, cont.

Table 1 , cont.

\begin{tabular}{|l|c|}
\hline \multicolumn{1}{|c|}{ Família/Espécie } & Coleta \\
\hline SAPINDACEAE & \\
Allophylus edulis Radlk. ex Warm. & 2896 \\
Allophylus sericeus Radlk. & 2979 \\
Cupania vernalis Cambess. & 2927 \\
Dilodendron bipinnatum Radlk. & 4294 \\
Matayba elaeagnoides Radlk. & 3057 \\
\hline SAPOTACEAE & \\
Chrysophyllum gonocarpum Engl. & 2942 \\
Pouteria sp. & 3049 \\
Pouteria sp.1 & 3010 \\
\hline SOLANACEAE & \\
Cestrum sp. & 2977 \\
Solanum argenteum Dunal ex Poir. & 3051 \\
Solanum cernuun Vell. & 2931 \\
Solanum granuloso-leprosum Dunal & 3047 \\
Solanum leucodendron Sendt. & 2983 \\
Solanum sp. & 3027 \\
\hline STERCULIACEAE & \\
Sterculia chicha A. St.-Hil. & 2967 \\
\hline SYMPLOCACEAE & \\
Symplocos pubescens Klotzsch ex Benth. & 2987 \\
\hline TILIACEAE & \\
Luehea grandiflora Mart. \& Zucc. & 3037 \\
\hline ULMACEAE & \\
Trema micrantha Blume & 3074 \\
\hline VERBENACEAE & \\
Aegiphila sellowiana Cham. & 2962 \\
Aloysia virgata Juss. & 3072 \\
Vitex montevidensis Cham. & 3073 \\
Vitex sellowiana Cham. & 3075 \\
\hline VOCHYSIACEAE & 3071 \\
Qualea jundiahy Warm. & 2928 \\
\hline Vochysia tucanorum Mart. & \\
\hline
\end{tabular}

As espécies que ocorreram na Mata da Pedreira e também nas áreas de maior similaridade, foram: Tapirira guianensis, Guatteria vilosissima, Rollinia silvatica, Xylopia sericea, Schefflera morototoni, Vernonia diffusa, Jacaranda macrantha, Sparattosperma leucanthum, Cassia ferrruginea, Copaifera langsdorffii, Croton floribundus, Andira fraxinifolia, Machaerium stipitatum, Platycyamus regnellii, Casearia decandra, Casearia sylvestris, Endlicheria paniculata, Nectandra lanceolata, Ocotea corymbosa, Ocotea odorifera, Cariniana estrellensis, Miconia cinnamomifolia, Trichilia pallida, Anadenanthera colubrina, Piptadenia gonoacantha, Siparuna arianeae, Maclura tinctoria, Guapira opposita, Prunus sellowii, Amaioua guianensis, Coutarea speciosa, Guettarda viburnoides, Cupania vernalis, Matayba elaeagnoides, Luehea grandiflora e Aegiphila sellowiana.

As áreas de maior similaridade com a Mata da Pedreira estão estabelecidas em altitudes acima de $500 \mathrm{~m}$, que, segundo Veloso (1991), são formações caracterizadas como Floresta Estacional Semidecidual Montana. Meira-Neto (1997), ao estudar a Mata da Silvicultura em Viçosa, Estado de Minas Gerais, estabeleceu uma comparação florística com dez florestas por meio de análise de agrupamentos, utilizando as espécies indicadoras de florestas de altitude dentre as espécies comuns entre a Mata da Silvicultura e as demais. O autor afirma que existe uma clara influência da vegetação arbórea montana na composição florística da área por ele

Quadro 2 - Índice de similaridade florística de Sorensen calculado entre o presente estudo e outros trabalhos realizados em Florestas Estacionais Semideciduais (F.E.S) e Florestas Ombrófilas Densas (FOMD) para espécies arbóreas, em que $\mathrm{N}$ = número de espécies, $\mathrm{EC}=$ espécies comuns em relação ao trabalho atual e IS = índice de similaridade

Table 2 - Floristic similarity index of SORENSEN, calculated between the present study and other works carried out on Semideciduous Seasonal Forests (F.E.S) and Thick Ombrofilous Forests (F.OM.D) for arboreal species, where: $N=$ number of species, EC = common species related to the present work, IS = similarity index

\begin{tabular}{|c|c|c|c|c|}
\hline Local & Tipologia florestal & $\mathrm{N}$ & $\mathrm{EC}$ & IS\% \\
\hline Mata da Silvicultura - Viçosa-MG (Meira-Neto, 1997) & F.E.S & 154 & 71 & 47,17 \\
\hline Vinhático - Parque Florestal Estadual do R. Doce, MG (Lopes, 1998) & F.E.S & 145 & 49 & 33,56 \\
\hline Serra de São José -Tiradentes, MG (Oliveira-Filho \& Machado, 1993) & F.E.S & 277 & 61 & 32,62 \\
\hline Rio Claro, SP (Pagano et al., 1987) & F.E.S & 201 & 50 & 28,73 \\
\hline Floresta Nacional do Rio Preto, ES (Salomão, 1998) & F.OM.D & 338 & 30 & 12,37 \\
\hline Poço Bonito - Lavras, MG (Oliveira-Filho et al., 1994) & F.E.S & 119 & 16 & 12,03 \\
\hline Volta Velha, SC (Negrelle, 1995) & F.OM.D & 196 & 19 & 11,07 \\
\hline Ubatuba, SP (Silva \& Leitão-Filho, 1982) & F.OM.D & 123 & 10 & 7,40 \\
\hline
\end{tabular}


estudada. A áre do presente estudo pertence a essa formação, que é bem típica da Zona da Mata de Minas Gerais. A similaridade entre a Mata da Pedreira e a Mata da Silvicultura foi a maior (48\%) das oito florestas comparadas.

A área estudada mostrou uma riqueza específica que merece atenção especial, visto que o banco genético ali estabelecido deve ser preservado, garantindo a tipologia da região para gerações futuras. Tal florística estabelece uma base para que se possa fazer o manejo com o intuito de preservar esse remanescente de Floresta Estacional Semidecidual da Zona da Mata de Minas Gerais.

\section{REFERÊNCIAS BIBLIOGRÁFICAS}

ALMEIDA, D. S. Florística e estrutura de um fragmento de Floresta Atlântica, no município de Juiz de Fora , Minas Gerais. 1996. 91 f. Dissertação (Mestrado em Ciência Florestal) - Universidade Federal de Viçosa, Viçosa, MG, 1996.

BARROSO, G. M. Sistemática das angiospermas do Brasil. Viçosa: UFV, 1984. 377 p.

CORREA, M. P. Dicionário das plantas úteis do Brasil. Rio de Janeiro: Ministério da Agricultura, IBDF, 1929.

CRONQUIST, A. The evolution and classification of flowering plants. New York: The New York Botanical Garden, 1988. 555 p.

LEITÃO FILHO, H. F. Aspectos taxonômicos das florestas do Estado de São Paulo. Silvicultura em São Paulo, v. 16, n. 1, p. 197-206, 1982.

LOPES, W. P. Florística e fitossociologia da vegetação arbórea na região do Vinhático - Parque Estadual do Rio Doce, Minas Gerais. 1998. 69 f. Dissertação (Mestrado em Ciência Florestal) - Universidade Federal de Viçosa, Viçosa-MG, 1998.

LORENZI, H. Árvores brasileiras: manual de identificação e cultivo de plantas arbóreas nativas do Brasil. Nova Odessa: Plantarum, 1992. 352 p.

MARANGON, L. C. Florística e fitossociologia de área de floresta estacional semidecidual visando dinâmica de espécies florestais arbóreas no município de Viçosa-MG. 1999. 139 f. Tese (Doutorado em Ecologia e Recursos Naturais) - Universidade Federal de São Carlos, São Carlos, 1999.

R. Árvore, Viçosa-MG, v.27, n.2, p.207-215, 2003
MARISCAL FLORES, E. J. Potencial produtivo e alternativas de manejo sustentável de um fragmento de Mata Atlântica secundária, município de Viçosa, Minas Gerais. 1993. 165 f. Dissertação (Mestrado em Ciência Florestal) - Universidade Federal de Viçosa, Viçosa-MG, 1993.

MEIRA-NETO, J. A. A. Estudos florísticos, estruturais e ambientais nos estratos arbóreos e herbáceo-arbustivo de uma floresta estacional semidecidual em Viçosa, MG. 1997. 154 f. Tese (Doutorado em Biologia) - Universidade Estadual de Campinas, Viçosa-MG, 1997.

MUELLER-DOMBOIS, D.; ELLEMBERG, H. Aims and methods of vegetation ecology. New York, John Wiley \& Sons, 1974. 547 p.

NEGRELLE, R. R. B. Composição florística, estrutura fitossociológica e dinâmica de regeneração da floresta Atlântica na reserva Volta Velha, mun. Itapoã, SC. 1995. 222 f. Tese (Doutorado em Ciências) - Universidade Federal de São Carlos, São Carlos, 1995.

OLIVEIRA FILHO, A. T.; MACHADO, J. N. M. Composição florística de uma floresta semidecídua montana, na serra de São José, Tiradentes, Minas Gerais. Acta Botânica Brasilica, v. 7, n. 2, p. 71-88, 1993.

OLIVEIRA FILHO, A. T.; SCOLFORO, J. R. S.; MELLO, J. M. Composição florística e estrutura comunitária de um remanescente de floresta semidecídua montana em Lavras, MG. Revista Brasileira de Botânica, v. 17, n. 2, p. 167$182,1994$.

PAGANO, S. N.; LEITÃO FILHO, H. F.; SHEPHERD, G. J. Estudo fitossociológico em mata mesófila semidecídua, no município de Rio Claro, SP. Revista Brasileira de Botânica, v. 10 , n. 1 , p. $37-48,1987$.

RESENDE, S. B. Estudo de crono-seqüência em Viçosa Minas Gerais. Viçosa. 1971. 71 f. Dissertação (Mestrado em Solos) - Universidade Federal de Viçosa, 1971.

REITZ, R.; KLEIN, R. M.; REIS, A. Projeto madeiras de Santa Catarina. Florianópolis: Lunardelli, 1979. 320 p.

RIZZINI, C. T. Árvores e madeiras úteis do Brasil, manual de dendrologia brasileira. São Paulo: Edgard Blücher, 1971. $126 \mathrm{p}$.

ROYAL BOTANIC GARDENS. Index Kewensis on compact disc-manual. Oxford: University Press, 1993. 67 p. 
SALOMÃO, A. L. F. Subsídios técnicos para elaboração do plano de manejo da floresta nacional do Rio Preto-ES. 1998. 151 f. Tese (Doutorado em Ciência Florestal) Universidade Federal de Viçosa, Viçosa-MG, 1998.

SILVA, A. F.; LEITÃO FILHO, H. F. Composição florística e estrutura de um trecho de Mata Atlântica de encosta no município de Ubatuba, SP. Revista Brasileira de Botânica, v. 5, p. 55-94, 1982.

VALVERDE, O. Estudo regional da Zona da Mata de Minas Gerais. Revista Brasileira de Geografia, v. 20, n. 1, p. 182. 1958 .
VELOSO, H. P.; GOES FILHO, L. Fitogeografia brasileira, classificação fisionômica ecológica da vegetação neotropical. Projeto RADAMBRASIL, Sér. Vegetação, Salvador: 1982, 80 p. (Boletim Técnico, 1)

VELOSO, H. P.; RANGEL FILHO, A. L. R.; LIMA, J. C. A. Classificação da vegetação brasileira adaptada a um sistema universal. São Paulo: Fundação Instituto Brasileiro de Geografia e Estatística - IBGE, 1991. 123 p.

VIEIRA, M. F.; CARVALHO-OKANO, R. M. Instruções básicas para coleta e remessa de planta para identificação. Viçosa: UFV, 1985. 11 p. (Informe Técnico, 53). 